\title{
A novel SCAR marker for detecting Psathyrostachys huashanica Keng chromatin introduced in wheat
}

\author{
W.L. Du, J. Wang, L.M. Wang, Y.H. Pang, J. Wu, J.X. Zhao, Q.H. Yang \\ and X.H. Chen
}

Key Laboratory of Genetic Engineering for Plant Breeding of Shaanxi Province, College of Agronomy, Northwest A\&F University, Yangling, Shaanxi, China

Corresponding author: X.H. Chen

E-mail: cxh2089@126.com

Genet. Mol. Res. 12 (4): 4797-4806 (2013)

Received November 30, 2012

Accepted June 10, 2013

Published October 18, 2013

DOI http://dx.doi.org/10.4238/2013.October.18.16

\begin{abstract}
In this study, we cloned and sequenced a 938-base pair polymorphic band, pHs27, in the tightly linked random amplified polymorphic DNA marker OPU10 and converted it into a sequencecharacterized amplified region (SCAR) marker referred to as RHS141, which was specific for the Ns genome of Psathyrostachys huashanica. A GenBank basic local alignment search tool search showed that the sequence of pHs27 had no primary sequence homology with known sequences, and Southern blotting confirmed this result. This SCAR marker was used to detect Ns genome chromatin in wheat, and it was successfully amplified in $P$. huashanica itself, a complete set of wheat- $P$. huashanica disomic addition lines (1Ns-7Ns), and undetermined homoeologous group addition lines. This SCAR marker will be a powerful tool for the marker-assisted selection of $P$. huashanica chromosome(s) in a wheat background, and it should also allow wheat breeders to screen for the excellent traits found in P. huashanica chromatin.
\end{abstract}

Key words: Marker-assisted selection; Psathyrostachys huashanica; RAPD; Repetitive sequence; SCAR; Wheat 


\section{INTRODUCTION}

The production and utilization of polymerase chain reaction (PCR)-based markers is effective for tracing specific alien chromatin or genes during the breeding process (Landjeva et al., 2007). Amplified fragment length polymorphism (AFLP) markers and random amplified polymorphic DNA (RAPD) markers have been used successfully in recent years to develop reliable sequence-tagged, sequence-characterized amplified region (SCAR) markers in crops. However, the development of sequence-specific primers for SCAR markers based on AFLP markers is not an efficient process in wheat (Shan et al., 1999). In contrast, RAPD has the advantages of being rapid, cost effective, and supportive of high levels of polymorphism, and it has been used for the identification of crops (Scheef et al., 2003).

Psathyrostachys huashanica Keng $(2 n=2 x=14$, NsNs $)$ is a perennial cross-pollinating plant that is found only on Huashan Mountain in the Shaanxi Province of China (Kuo, 1987) and is a potentially useful germplasm source in China. It is characterized by high resistance to cold, salinity, drought, and barren conditions, and it also exhibits a dwarfed stature, early maturity, and resistance to scab, stripe rust, take-all, and powdery mildew (Zhao et al., 2010; Wang et al., 2011).

In order to transfer desirable traits from $P$. huashanica into wheat, our research team successfully developed a complete set of wheat- $P$. huashanica disomic addition lines (1Ns-7Ns) and undetermined homoeologous group addition lines, which were supported by cytogenetic, genomic in situ hybridization (GISH), and molecular marker expressed sequence tag-simple sequence repeat (EST-SSR) and EST-sequence tagged site (STS) analysis (Du et al., 2013a,b,c,d; 1Ns, 2Ns, and 3Ns unpublished). However, no specific molecular markers have been reported to identify the Ns genome from $P$. huashanica. Therefore, new and specific molecular markers are urgently needed to rapidly and accurately detect $P$. huashanica chromosome(s). In this study, we isolated a novel repetitive DNA fragment from the Ns genome of $P$. huashanica using RAPD analysis, which we converted into a fast and reliable SCAR marker specifically for the Ns genome of $P$. huashanica. The main objective of this study was to develop and characterize a SCAR marker that could be used to distinguish wheat lines carrying chromosome(s) derived from P. huashanica.

\section{MATERIAL AND METHODS}

\section{Plant materials}

The genomic materials used in this study are shown in Table 1, which also includes the controls used in the RAPD analysis. The wheat cultivar ' 7182 ' and P. huashanica were used as parental materials for intergeneric hybridization, and 22 addition line plants were used to validate the SCAR marker RHS141, as shown in Table 2. These specimens were deposited at the Key Laboratory of Genetic Engineering for Plant Breeding of Shaanxi Province, College of Agronomy, Northwest A\&F University, Shaanxi, China.

\section{DNA extraction and RAPD assay}

The total genomic DNA was extracted from fresh leaves using the modified cetyl 
trimethyl ammonium bromide (CTAB) method (Cota-Sánchez et al., 2006). A RAPD assay was used to screen 200 random decamer primers. DNA amplification was performed following the procedure described by (Wu et al., 2010). The amplified products were fractionated on a $1.0 \%$ agarose gel using $1 \mathrm{X}$ Tris-acetate-ethylenediaminetetraacetic acid (TAE) buffer. The gels were stained with ethidium bromide (EB) and visualized using an automatic gel imaging analysis system.

Table 1. Study species, ploidy level, genomic constitution, and origin.

\begin{tabular}{|c|c|c|c|c|}
\hline & Species & Ploidy & Genome & Origin \\
\hline Common wheat cultivars & 7182 (Triticum aestivum L.) & $6 \mathrm{x}$ & AABBDD & Our research group \\
\hline \multirow[t]{5}{*}{ Rare species } & Psathyrostachys huashanica Keng & $2 \mathrm{x}$ & NsNs & \\
\hline & Triticum amyleum $\mathrm{L}$. & $2 \mathrm{x}$ & AA & \\
\hline & Triticum dicoccoides & $4 \mathrm{x}$ & AABB & \\
\hline & Triticum araraticum Jakubz. & $4 \mathrm{x}$ & AAGG & \\
\hline & Triticum zhukovskyi Men. et Er. & $6 \mathrm{x}$ & AAAAGG & \\
\hline \multirow[t]{21}{*}{ Wild relative species } & Aegilops markgrafii (Greuter) Hammer & $2 \mathrm{x}$ & $\mathrm{CC}$ & Chinese Academy of Agricultural Sciences \\
\hline & Aegilops caudata $\mathrm{L}$. & $2 \mathrm{x}$ & $\mathrm{CC}$ & \\
\hline & Aegilops tauschii (Coss.) Schmal. & $2 \mathrm{x}$ & DD & \\
\hline & Thinopyrum elongatum & $2 \mathrm{x}$ & $\mathrm{EE}$ & \\
\hline & Hordeum violaceum & $2 \mathrm{x}$ & $\mathrm{HH}$ & \\
\hline & Hordeum vulgare $\mathrm{L}$. & $2 \mathrm{x}$ & II & \\
\hline & Crithopsis delileana (Schult) Roshev & $2 \mathrm{x}$ & KK & \\
\hline & Aegilops comosa Sm. in Sibth. \& Sm. & $2 \mathrm{x}$ & $\mathrm{MM}$ & \\
\hline & Agropyron cristatum Gaertn. & $6 \mathrm{x}$ & РРРРРР & \\
\hline & Eremopyrum orientale & $4 \mathrm{x}$ & $\mathrm{B}^{\prime} \mathrm{B}^{\prime} \mathrm{C}^{\prime} \mathrm{C}^{\prime}$ & \\
\hline & Triticum timopheevii Zhuk. & $4 \mathrm{x}$ & AtAtGG & \\
\hline & Secale cereale $\mathrm{L}$. & $2 \mathrm{x}$ & $\mathrm{RR}$ & \\
\hline & Aegilops speltoides Tausch & $2 \mathrm{x}$ & SS & \\
\hline & Roegneria ciliaris (trin) Nevski & $4 \mathrm{x}$ & SSYY & \\
\hline & Elymus rectisetus & $6 \mathrm{x}$ & SSYYWW & \\
\hline & Pseudoroegneria strigosa A. Love & $2 \mathrm{x}$ & StSt & \\
\hline & Roegneria grandiglumis Keng & $6 \mathrm{x}$ & StStPPYY & \\
\hline & Aegilops umbellulata Zhuk. & $2 \mathrm{x}$ & UU & \\
\hline & Haynaldia villosa (L.) Schur. & $2 x$ & VV & \\
\hline & Australopyrum coinitum retrofractum & $2 \mathrm{x}$ & WW & \\
\hline & Roegneria kamoji ohwi & $2 \mathrm{x}$ & YY & \\
\hline
\end{tabular}

\begin{tabular}{|c|c|c|c|c|c|c|c|}
\hline Plant code & $2 n$ & $\begin{array}{l}\text { Chromosome } \\
\text { composition }\end{array}$ & $\begin{array}{l}\text { Homoeologous } \\
\text { group }\end{array}$ & $\begin{array}{l}\text { Plant } \\
\text { code }\end{array}$ & $2 n$ & $\begin{array}{l}\text { Chromosome } \\
\text { composition }\end{array}$ & $\begin{array}{l}\text { Homoeologous } \\
\text { group }\end{array}$ \\
\hline P. huashanica & 14 & $14 \mathrm{Ns}$ & & $18-1-1$ & 44 & $42 \mathrm{~W}+2 \mathrm{Ns}$ & - \\
\hline 7182 & 42 & $42 \mathrm{~W}$ & & $21-4$ & 43 & $42 \mathrm{~W}+1 \mathrm{Ns}$ & - \\
\hline $12-3$ & 44 & $42 \mathrm{~W}+2 \mathrm{Ns}$ & 1 & $23-2-1$ & 44 & $42 \mathrm{~W}+2 \mathrm{Ns}$ & - \\
\hline $3-6-4-1$ & 44 & $42 \mathrm{~W}+2 \mathrm{Ns}$ & 2 & $24-5$ & 44 & $42 \mathrm{~W}+2 \mathrm{Ns}$ & - \\
\hline $22-2$ & 44 & $42 \mathrm{~W}+2 \mathrm{Ns}$ & 3 & $25-4-4$ & 44 & $42 \mathrm{~W}+2 \mathrm{Ns}$ & - \\
\hline $24-6-3$ & 44 & $42 \mathrm{~W}+2 \mathrm{Ns}$ & 4 & $27-4-4$ & 43 & $42 \mathrm{~W}+1 \mathrm{Ns}$ & - \\
\hline $3-8-10-2$ & 44 & $42 \mathrm{~W}+2 \mathrm{Ns}$ & 5 & $28-1-2$ & 43 & $42 \mathrm{~W}+1 \mathrm{Ns}$ & - \\
\hline $59-11$ & 44 & $42 \mathrm{~W}+2 \mathrm{Ns}$ & 6 & $30-2$ & 44 & $42 \mathrm{~W}+2 \mathrm{Ns}$ & - \\
\hline $2-1-6-3$ & 44 & $42 \mathrm{~W}+2 \mathrm{Ns}$ & 7 & $31-6$ & 44 & $42 \mathrm{~W}+2 \mathrm{Ns}$ & - \\
\hline $14-7$ & 44 & $42 \mathrm{~W}+2 \mathrm{Ns}$ & - & $33-1-10$ & 44 & $42 \mathrm{~W}+2 \mathrm{Ns}$ & - \\
\hline $15-9$ & 44 & $42 \mathrm{~W}+2 \mathrm{Ns}$ & - & $34-8-6$ & 43 & $42 \mathrm{~W}+1 \mathrm{Ns}$ & - \\
\hline $17-7$ & 43 & $42 \mathrm{~W}+1 \mathrm{Ns}$ & - & $38-2-4$ & 43 & $42 \mathrm{~W}+1 \mathrm{Ns}$ & - \\
\hline
\end{tabular}

$(-)=$ undetermined homoeologous groups. Ns and W were representives of Psathyrostachys huashanica and wheat chromosomes, respectively. 


\section{Cloning and sequence analysis}

P. huashanica-diagnostic RAPD bands were excised from $1 \%$ agarose gels using a gel extraction kit. The purified products were cloned into the pMD19-T vector and transformed into Escherichia coli DH5a competent cells by heat shock transformation. Plasmids from randomly selected white colonies were extracted using a plasmid kit. DNA sequencing was performed at Sangon Biotech (Shanghai, China). The plasmid construction was confirmed by enzyme digestion and DNA sequencing. The sequences were submitted to the National Center for Biotechnology Information (NCBI) network service to screen for the nonexistence of similar sequences or low similarity sequences based on comparisons. The nucleotide sequences that were unique to the Ns genome were submitted to GenBank and registered under the accession number HR614226.

\section{Southern blot hybridization}

The probe was labeled using a digoxigenin (DIG) High Prime DNA Labeling \& Detection Starter Kit (Roche, company, Germany) and hybridized overnight at $37^{\circ} \mathrm{C}$. The membrane transfer and washing procedures have been described previously (Wu et al., 2010). Images were acquired using an automatic gel imaging analysis system.

\section{SCAR primer design and specific amplification in various genomes}

Based on the nucleotide sequences of the RAPD products, a pair of SCAR marker primers was designed and synthesized for the specific amplification of loci identified by previous RAPD markers in various genomes (Table 3). The primers were used to amplify the specific marker under the following conditions: $2 \mu \mathrm{L} 10 \mathrm{X}$ polymerase chain reaction (PCR) buffer, $2 \mu \mathrm{L}$ primer $(2.5 \mathrm{mM}), 2 \mu \mathrm{L}$ DNA template $(50-100 \mathrm{ng} / \mu \mathrm{L}), 1.6 \mu \mathrm{L}$ dNTPs $(2.5 \mathrm{mM})$, $1.6 \mu \mathrm{L} \mathrm{MgCl}_{2}(2.5 \mathrm{M}), 0.2 \mu \mathrm{L}$, Taq polymerase $(5 \mathrm{U} / \mu \mathrm{L})$, and $10.6 \mu \mathrm{L} \mathrm{ddH}_{2} \mathrm{O}$. The amplification procedure comprised an initial denaturation at $94^{\circ} \mathrm{C}$ for $4 \mathrm{~min} ; 40$ cycles at $94^{\circ} \mathrm{C}$ for 50 $\mathrm{s}, 60^{\circ} \mathrm{C}$ for $50 \mathrm{~s}$, and $72^{\circ} \mathrm{C}$ for $1 \mathrm{~min}$; and a final extension at $72^{\circ} \mathrm{C}$ for $10 \mathrm{~min}$. The amplified products were resolved by electrophoresis using $1 \%$ agarose gels, as described earlier.

Table 3. Characteristics of the SCAR marker developed for the Ns genome of Psathyrostachys huashanica.

\begin{tabular}{lcclcr}
\hline Marker & Type & Accession No. & Primer sequence $\left(5^{\prime}-3^{\prime}\right)$ & Size $($ bp $)$ & Annealing temperature $\left({ }^{\circ} \mathrm{C}\right)$ \\
\hline OPU10 & RAPD & & ACCTCGGCAC & 938 & 34 \\
RHS141 & SCAR & HR614226 & F: CTCGGCACCATAAACTAT & 938 & 60 \\
& & R: CTCGGCACTAGAGGAAAC & & \\
\hline
\end{tabular}

\section{Verifying the SCAR marker in wheat-P. huashanica addition lines}

The SCAR marker RHS141 was used to test the validity of the molecular marker in the wheat cultivar '7182', P. huashanica, a complete set of wheat- $P$. huashanica disomic addition lines (1Ns-7Ns), and undetermined homoeologous group addition lines. The conditions for the SCAR reactions were the same as those used for the specific amplifications in various genomes. 


\section{RESULTS}

\section{Species diagnosis and RAPD markers}

After screening 200 RAPD primers, we selected 32 primers that produced distinct $P$. huashanica genome-specific bands. The RAPD primer OPU10 diagnostic band was clearly observed in P. huashanica, and it was missing from the genomes of other species (Figure 1), which confirmed that primer OPU10 was a good candidate for the specific detection of the $P$. huashanica genome. The other RAPD primer diagnostic bands are not shown.

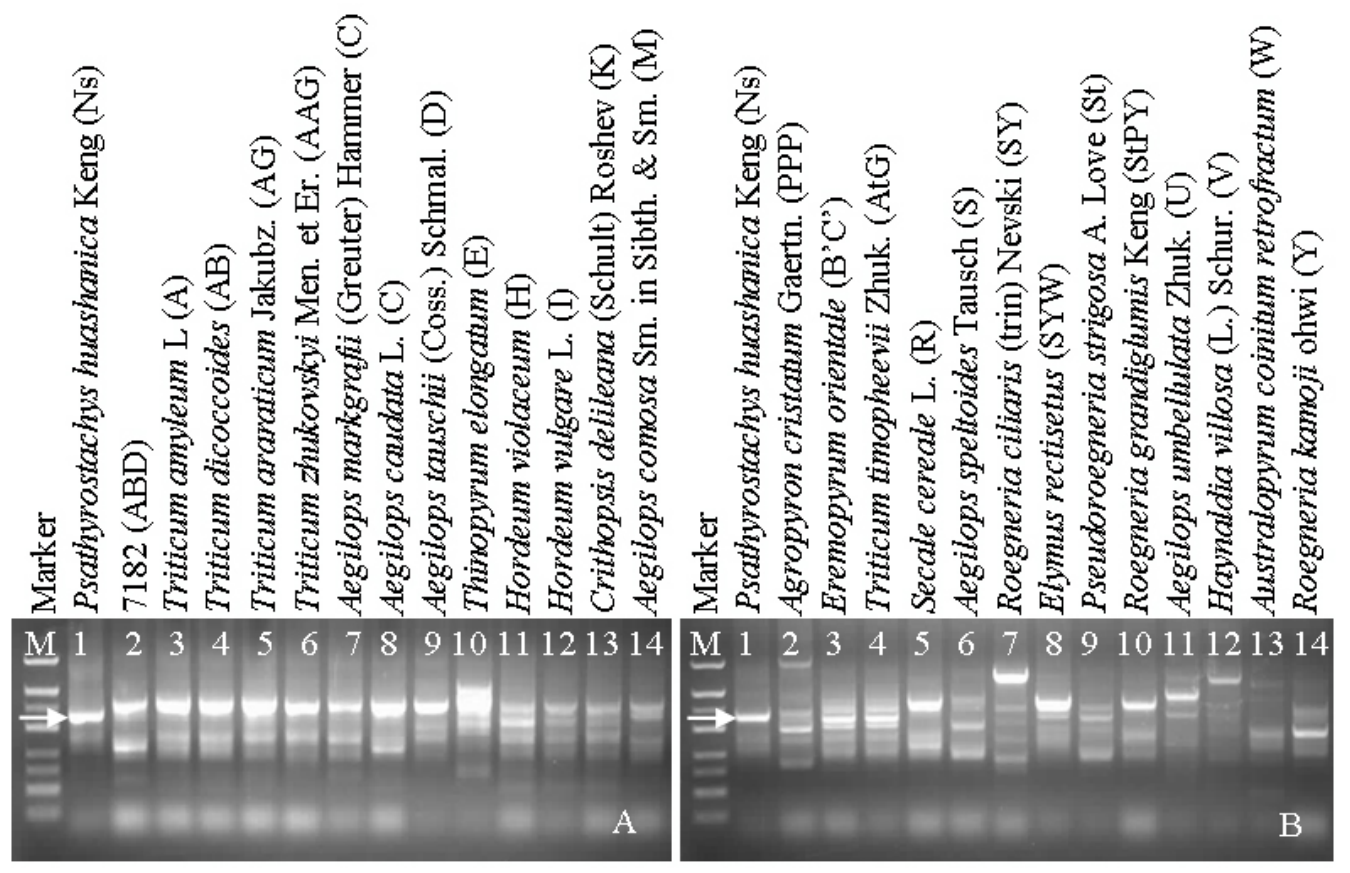

Figure 1. Psathyrostachys huashanica genome-specific RAPD fragments isolated by amplification using the OPU10 decamer as a primer in: A. Lane $M=$ marker; lane $1=\mathrm{Ns}$; lane $2=\mathrm{ABD}$; lane $3=\mathrm{A}$; lane $4=\mathrm{AB}$; lane $5=$ $\mathrm{AG}$; lane $6=\mathrm{AAG}$; lane $7=\mathrm{C}$; lane $8=\mathrm{C}$; lane $9=\mathrm{D}$; lane $10=\mathrm{E}$; lane $11=\mathrm{H}$; lane $12=\mathrm{I}$; lane $13=\mathrm{K}$; lane 14 $=\mathrm{M}$. B. Lane $M=$ marker; lane $1=\mathrm{Ns}$; lane $2=\mathrm{PPP}$; lane $3=\mathrm{B}{ }^{\prime} \mathrm{C}$ '; lane $4=\mathrm{AtG}$; lane $5=\mathrm{R}$; lane $6=\mathrm{S} ;$ lane $7=$ $\mathrm{SY}$; lane $8=\mathrm{SYW}$; lane $9=\mathrm{St}$; lane $10=\mathrm{StPY}$; lane $11=\mathrm{U}$; lane $12=\mathrm{V}$; lane $13=\mathrm{W}$, and lane $14=\mathrm{Y}$ genomes. The arrows indicate the diagnostic amplification product of $P$. huashanica.

\section{Sequence analysis}

The P. huashanica genome-specific RAPD PCR product was cloned, sequenced, and designated pHs27, the full length of which was $938 \mathrm{bp}$ (Figure 2). It had no special characteristics such as long terminal repeats, inverted repeats, or palindromes. Further sequence homology searches were conducted using the nucleotide basic local alignment search tool 
(BLASTn) and translated BLAST (BLASTx) programs. The results showed that the sequence of pHs27 shared no similarity with sequences in the NCBI database. We hypothesized that $\mathrm{pHs} 27$ was a new $P$. huashanica repetitive DNA sequence.

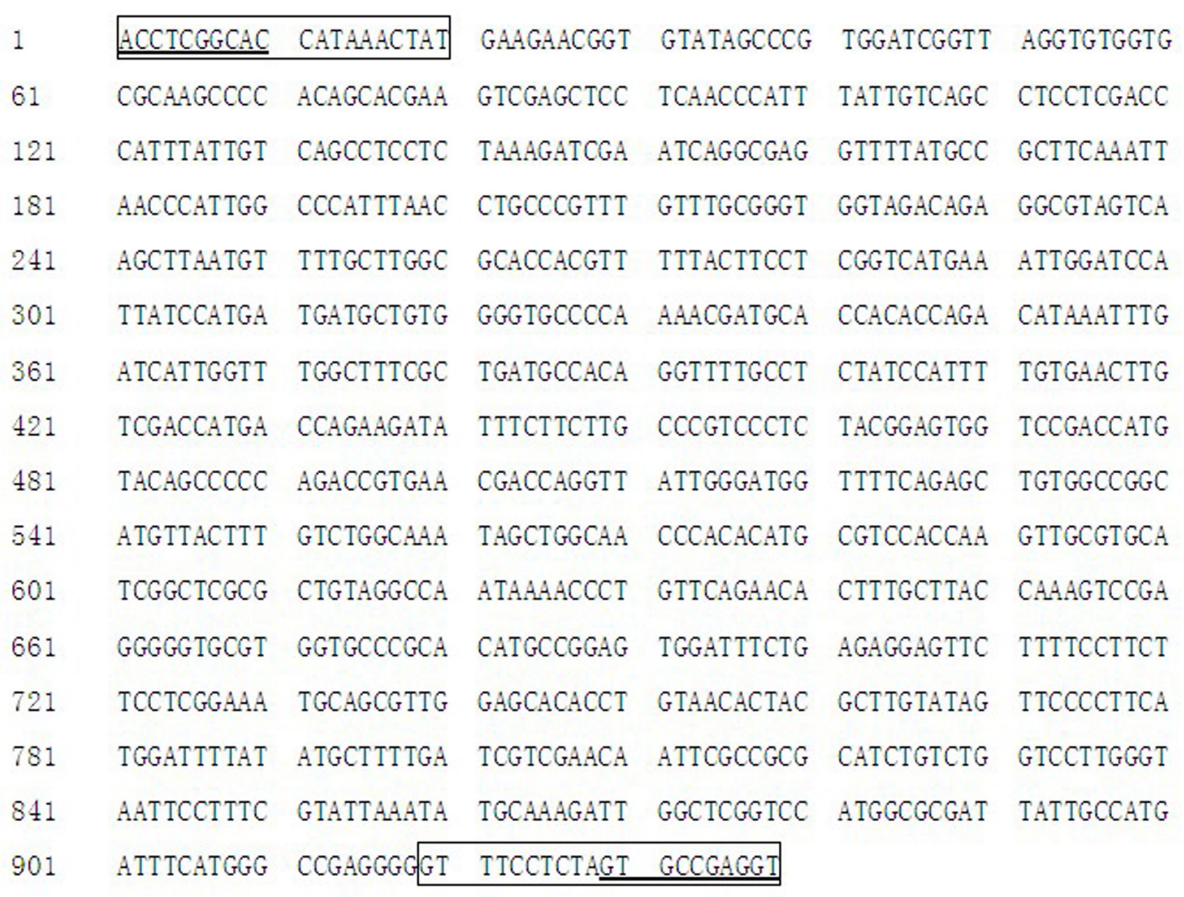

Figure 2. Nucleotide sequence of pHs27, which has an accession No. of HR614226. The primer regions are marked while the underlined and boxed regions indicate the RAPD primer sequence and SCAR primer sequence, respectively.

\section{Southern blot hybridization}

Southern blot hybridization detected a strong, smeared signal of the probe pHs27 (Figure 3). A prominent band corresponded to the Ns genome of the P. huashanica species, which also suggested that the sequence was dispersed in the Ns genome of $P$. huashanica. However, the signal was not present in the other genomes, which was consistent with the PCR amplification results. These data confirmed that the pHs 27 clone is a novel, Ns genomespecific DNA sequence in $P$. huashanica.

\section{Verification of the SCAR marker RHS141 in various genomes}

The polymorphic SCAR marker RHS141 was amplified in various genomes. However, it was only present in the Ns genome of P. huashanica, and it was absent from all of the other genomes (Figure 4). This specificity of the SCAR marker will provide valuable information for tracing $P$. huashanica chromatin in a wheat background. 


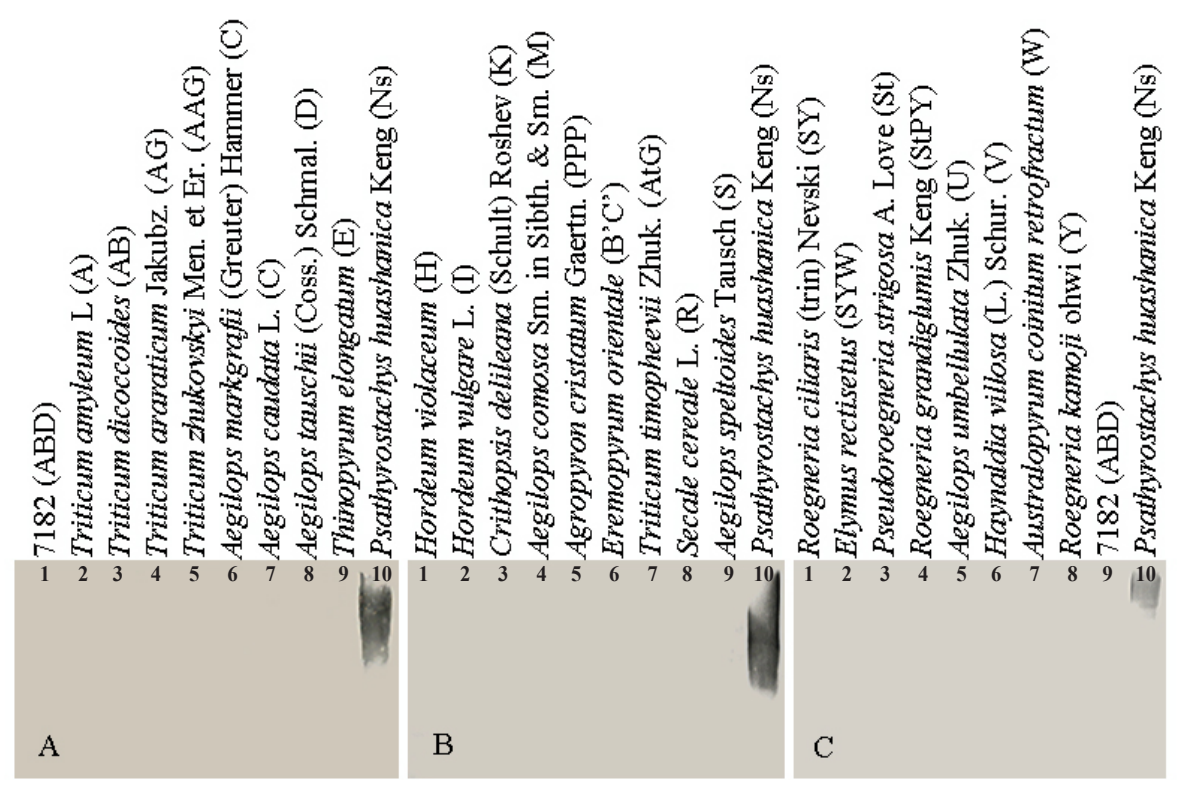

Figure 3. Southern hybridization using $\mathrm{pHs} 27$ as a probe with a range of total DNA samples. A. Lane $1=\mathrm{ABD}$; lane $2=\mathrm{A}$; lane $3=\mathrm{AB}$; lane $4=\mathrm{AG}$; lane $5=\mathrm{AAG}$; lane $6=\mathrm{C}$; lane $7=\mathrm{C}$; lane $8=\mathrm{D}$; lane $9=\mathrm{E}$; lane $10=\mathrm{Ns}$. B. Lane $1=\mathrm{H}$; lane $2=\mathrm{I}$; lane $3=\mathrm{K}$; lane $4=\mathrm{M}$; lane $5=\mathrm{PPP}$; lane $6=\mathrm{B}$ 'C'; lane $7=\mathrm{AtG}$; lane $8=\mathrm{R}$; lane 9 $=\mathrm{S}$; lane $10=\mathrm{Ns}$. C. Lane $1=\mathrm{SY}$; lane $2=\mathrm{SYW}$; lane $3=\mathrm{St}$; lane $4=\mathrm{StPY}$; lane $5=\mathrm{U}$; lane $6=\mathrm{V}$; lane $7=\mathrm{W}$; lane $8=\mathrm{Y}$; lane $9=\mathrm{ABD}$; lane $10=$ Ns. All DNAs were digested with HindIII.

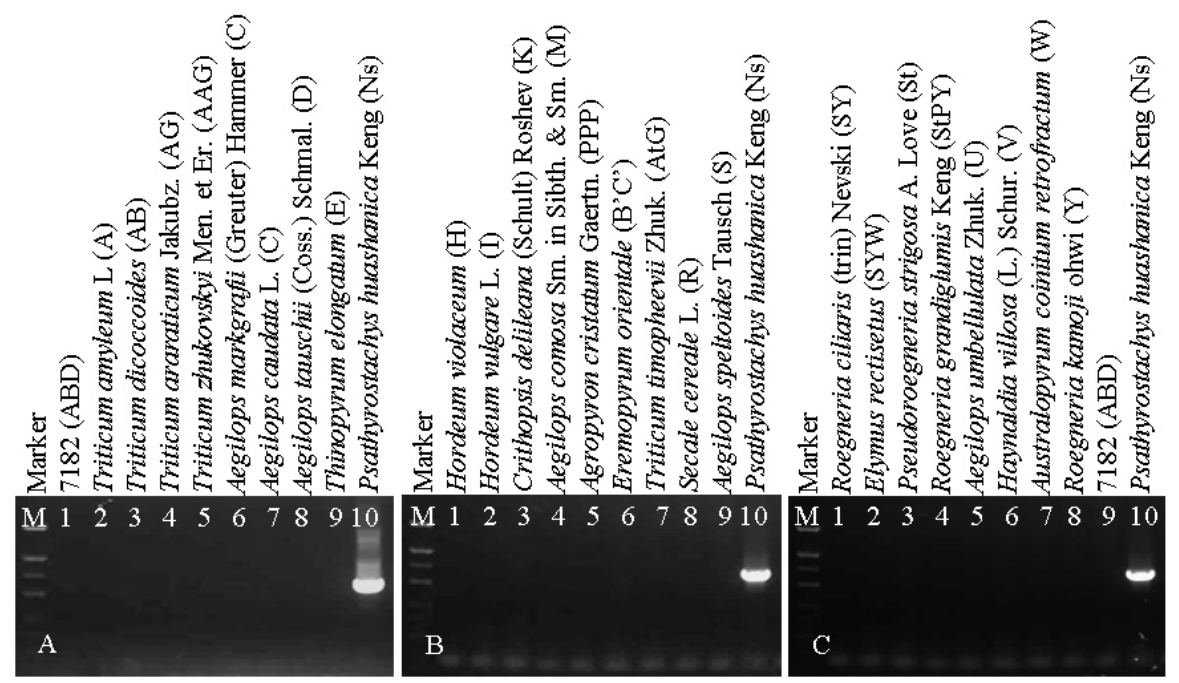

Figure 4. Validation of the SCAR marker RHS141 in the genomes of various species. A. Lane $M=$ marker; lane 1 $=\mathrm{ABD}$; lane $2=\mathrm{A}$; lane $3=\mathrm{AB}$; lane $4=\mathrm{AG}$; lane $5=\mathrm{AAG}$; lane $6=\mathrm{C}$; lane $7=\mathrm{C}$; lane $8=\mathrm{D} ;$ lane $9=\mathrm{E}$; lane $10=\mathrm{Ns}$. B. Lane $M=$ marker; lane $1=\mathrm{H}$; lane $2=\mathrm{I}$; lane $3=\mathrm{K}$; lane $4=\mathrm{M}$; lane $5=\mathrm{PPP}$; lane $6=\mathrm{B}^{\prime} \mathrm{C}^{\prime}$; lane 7 $=\mathrm{AtG}$; lane $8=\mathrm{R}$; lane $9=\mathrm{S}$; lane $10=\mathrm{Ns}$. C. Lane $M=$ marker; Lane $1=\mathrm{SY}$; lane $2=\mathrm{SYW}$; lane $3=\mathrm{St}$; lane 4 $=\mathrm{StPY}$; lane $5=\mathrm{U}$; lane $6=\mathrm{V}$; lane $7=\mathrm{W}$; lane $8=\mathrm{Y}$; lane $9=\mathrm{ABD}$; lane $10=\mathrm{Ns}$. 


\section{Validation of the SCAR marker RHS141 in wheat-P. huashanica addition lines}

The specific Ns genome SCAR marker RHS141 produced a very intense band at 938 bp, which was absent in the female parent of 7182. Moreover, it was present in wheat- $P$. huashanica disomic addition lines carrying $P$. huashanica chromosomes $1 \mathrm{Ns}, 2 \mathrm{Ns}, 3 \mathrm{Ns}$, $4 \mathrm{Ns}$, $5 \mathrm{Ns}, 6 \mathrm{Ns}$, and $7 \mathrm{Ns}$, and it was also present in $P$. huashanica addition lines carrying undetermined $P$. huashanica chromosomes (Figure 5A and B). This was a fast and robust assay for tracking the presence of $P$. huashanica chromatin in wheat.

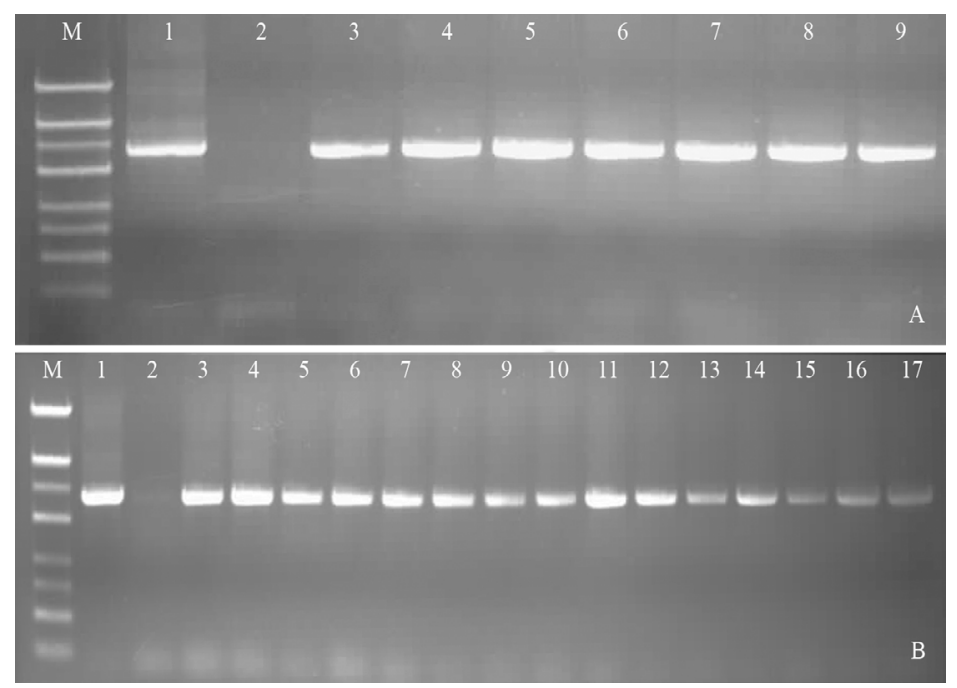

Figure 5. Identification of Psathyrostachys huashanica chromatin using the SCAR marker. RHS141 amplified specific bands in seven wheat- $P$. huashanica disomic addition lines (1Ns-7Ns) and undetermined homoeologous group addition lines. A. Lane $M=$ marker; lane $1=P$. huashanica; lane $2=7182$; lanes $3-9=$ seven wheat- $P$. huashanica disomic addition lines $(1 \mathrm{Ns}-7 \mathrm{Ns})$. B. Lane $M=$ marker; lane $1=P$. huashanica; lane $2=7182$; lanes 3-17 = undetermined homoeologous group addition lines.

\section{DISCUSSION}

The identification of exogenous chromosome(s) or chromosome segments is very important after alien species are introduced successfully into wheat. The question of how accurately and effectively alien differentiate chromosomes and fragment is of great theoretical and practical significance because it could provide a means to transfer exotic genes into cultivated wheat, develop translocation lines, and function as a bridging material for breeding (Hernández et al., 1999). RAPD is a simple and inexpensive technique that may facilitate the analysis of a large proportion of the genome and the identification of bands suitable for conversion to SCAR, which makes it suitable for marker-assisted selection (Hernández et al., 2001).

$P$. huashanica has attracted considerable attention among wheat breeders because of its excellent agronomic traits. Thus, many researchers have successfully transferred useful traits from $P$. huashanica into hexaploid wheat via intergeneric hybridization (Chen et al., 1991; Kang et al., 2008). Therefore, the development of a fast and reliable technique for iden- 
tifying $P$. huashanica chromatin in a wheat background is very important. We used the specific SCAR marker RHS141 of $P$. huashanica as a practical tool for the detection of the alien chromatin of $P$. huashanica in a wheat background (Figure 5A and B). Our results suggest that it is possible to use the marker as a specific molecular marker for the chromosome(s) of $P$. huashanica. In addition, the unique Ns genome SCAR marker of $P$. huashanica could be used during marker-assisted selection to study genome evolution in wheat-P. huashanica offspring.

Identifying and cloning genome-specific DNA segments is valuable for determining the provenance, evolution, and source of alien hereditary substances during distant hybridization breeding (Wei and Wang, 1995; Zhang et al., 1998). RAPD markers usually display high polymorphism in alien species; therefore, they are useful tools for genetic diversity studies (Guadagnuolo et al., 2001). However, there have been no previous reports of a specific SCAR marker for the Ns genome based on a RAPD primer that could be used to detect the presence of $P$. huashanica chromatin in a common wheat background. In this study, we developed the $P$. huashanica genome-specific SCAR marker RHS141 and used it to screen test materials. We successfully identified those that contained $P$. huashanica chromatin. Our experimental data suggest that this pair of primers can be used to detect the presence of $P$. huashanica chromatin in a wheat background. In summary, the Ns genome-specific SCAR marker RHS141 will be particularly useful during the early stages of breeding when selecting hybrids between wheat and $P$. huashanica. This will provide an efficient alternative to laborious and time-consuming genetic testing, which are currently a prerequisite for breeding.

\section{ACKNOWLEDGMENTS}

Research supported by the Ministry of Science and Technology of the People's Republic of China (\#2011AA10010203), the Northwest A\&F University (\#Z109021002 and \#A212020716), and the Tang Zhong-Ying Breeding Funding Project of the Northwest A\&F University, which are gratefully appreciated. The authors would like to thank Dr. Duncan E. Jackson for useful advice and English language editing of the manuscript.

\section{REFERENCES}

Chen SY, Zhang AJ and Fu J (1991). The hybridization between Triticum aestivum and Psathyrotachys huashanica. Acta Genet. Sin. 18: 508-512.

Cota-Sánchez JH, Remarchuk K and Ubayasena K (2006). Ready-to-use DNA extracted with a CTAB method adapted for herbarium specimens and mucilaginous plant tissue. Plant Mol. Biol. Rep. 24: 161-167.

Du WL, Wang J, Lu M, Sun SG, et al. (2013a). Characterization of a wheat-Psathyrostachys huashanica Keng 4Ns disomic addition line for enhanced tiller numbers and stripe rust resistance. Planta DOI 10.1007/s00425-013-1957-2.

Du WL, Wang J, Lu M, Sun SG, et al. (2013b). Molecular cytogenetic identification of a wheat-Psathyrostachys huashanica Keng 5Ns disomic addition line with stripe rust resistance. Mol. Breed. 31: 879-888.

Du WL, Wang J, Pang YH, Li YL, et al. (2013c). Isolation and characterization of a Psathyrostachys huashanica Keng 6Ns chromosome addition in common wheat. PLoS One 8: e53921.

Du WL, Wang J, Wang LM, Zhang J, et al. (2013d). Development and characterization of a Psathyrostachys huashanica Keng 7Ns chromosome addition line with leaf rust resistance. PLoS One 8: e70879.

Guadagnuolo R, Bianchi DS and Felber F (2001). Specific genetic markers for wheat, spelt, and four wild relatives: comparison of isozymes, RAPDs, and wheat microsatellites. Genome 44: 610-621.

Hernández P, Martín A and Dorado G (1999). Development of SCARs by direct sequencing of RAPD products: a practical tool for the introgression and marker-assisted selection of wheat. Mol. Breed. 5: 245-253.

Hernández P, Dorado G, Prieto P, Giménez MJ, et al. (2001). A core genetic map of Hordeum chilense and comparisons 
with maps of barley (Hordeum vulgare) and wheat (Triticum aestivum). Theor. Appl. Genet. 102: 1259-1264.

Kang HY, Zhang HQ, Fan X and Zhou YH (2008). Morphological and cytogenetic studies on the hybrid between bread wheat and Psathyrostachys huashanica Keng ex Kuo. Euphytica 162: 441-448.

Kuo PC (1987). Flora Reipublicae Popularis Sinicae. Science Press, Beijing, 51-104.

Landjeva S, Korzun V and Börner A (2007). Molecular markers: actual and potential contributions to wheat genome characterization and breeding. Euphytica 156: 271-296.

Scheef EA, Casler MD and Jung G (2003). Development of species-specific SCAR markers in bentgrass. Crop Sci. 43: 345-349.

Shan X, Blake TK and Talbert LE (1999). Conversion of AFLP markers to sequence-specific PCR markers in barley and wheat. Theor. Appl. Genet. 98: 1072-1087.

Wang Y, Xie Q, Yu K, Poysa V, et al. (2011). Development and characterization of wheat-Psathyrostachys huashanica partial amphiploids for resistance to stripe rust. Biotechnol. Lett. 33: 1233-1238.

Wei JZ and Wang RR (1995). Genome- and species-specific markers and genome relationships of diploid perennial species in Triticeae based on RAPD analyses. Genome 38: 1230-1236.

Wu M, Zhang JP, Wang JC, Yang XM, et al. (2010). Cloning and characterization of repetitive sequences and development of SCAR markers specific for the P genome of Agropyron cristatum. Euphytica 172: 363-372.

Zhang XY, Dong YS, Li P and Wang RRC (1998). Distribution of E and St-specific RAPD fragments in few genomes of Triticea. Acta Genet. Sin. 25: 131-141.

Zhao JX, Ji WQ, Wu J, Chen XH, et al. (2010). Development and identification of a wheat-Psathyrostachys huashanica addition line carrying HMW-GS, LMW-GS and gliadin genes. Genet. Resour. Crop Evol. 57: 387-394. 